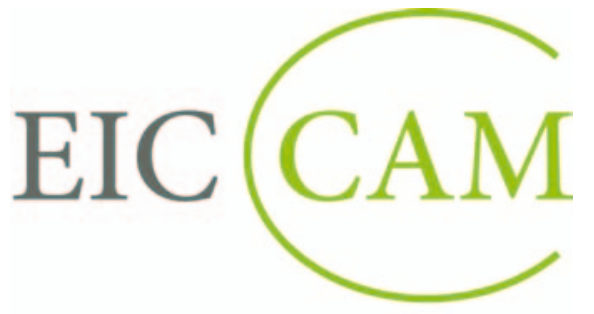

European Information Centre for

Complementary \& Alternative Medicine

«Komplementäre und Alternative Medizin ist nicht evidenzbasiert» - das wird bei Gesprächen mit politischen Entscheidungsträgern unablässig wiederholt. Deshalb ist es dringend notwendig, die Botschaft weiterzugeben, dass Komplementär- und Alternativmedizin (CAM) über viele hochwertige Forschungsergebnisse verfügt und darüber hinaus erhebliches Potenzial hat, einen wichtigen Beitrag zur Verbesserung der Gesundheitsversorgung in der EU zu leisten.

Der Informationsstand über die wissenschaftlichen Grundlagen von CAM ist wesentlich geringer als ihre zunehmende Nutzung durch die EUBürger. Um diese Informationslücke zu schliessen, wurde das European Information Center for Complementary and Alternative Medicine (EICCAM) mit dem Ziel der Bereitstellung und Verbreitung verständlicher, objektiver und qualitativ hochwertiger Informationen über Sicherheit, Wirksamkeit und Effizienz von CAM gegründet.

Die Ergebnisse der Forschung im Bereich von CAM werden in der Regel nur in wissenschaftlichen Zeitschriften publiziert und zwischen

\title{
European Information Center for Complementary and Alternative Medicine (EICCAM) gestartet
}

Wissenschaftlern kommuniziert unter Nutzung wissenschaftlicher Terminologie. Die daraus entstehende Mitteilungslücke ist ein Hindernis in der weiteren Verbreitung, Akzeptanz und Nutzung von CAM.

Deshalb ist es das zentrale Ziel von EICCAM, die vorhandenen wissenschaftlichen Informationen über CAM zu erfassen und regelmässig zu aktualisieren. Sie werden zusammengefasst und in einer verständlichen Form für interessierte Laien und die Öffentlichkeit aufbereitet. Die Zusammenstellung der Informationen erfolgt unabhängig, umfassend, qualitativ hochwertig und verständlich, um die Entscheidungsfindung von Politikern und Gesetzgebern und anderen wichtigen Gruppen im Gesundheitswesen $\mathrm{zu}$ erleichtern.

Das Zentrum ist mit der wissenschaftlichen Gemeinschaft und mit dem CAM-Repräsentanten vernetzt. Er beaufsichtigt die Organisation von oder die Teilnahme an wissenschaftlichen CAM-Veranstaltungen.

EICCAM wurde als Public-UtilityStiftung nach belgischem Recht gegründet, mit einem Management
Board und einem Scientific Board. Beide Beiräte entscheiden gemeinsam über Aktionen und Aktivitäten.

Der wissenschaftliche Beirat wählt die ein- und ausgehenden Informationen aus und sorgt für Qualität und Unabhängigkeit. Dem derzeitigen wissenschaftlichen Beirat gehören renommierte Wissenschaftler an, die an europäischen Universitäten entweder im CAM-Bereich forschen oder über das entsprechende Know-how verfügen. Es wurden bereits verschiedene «EICCAM Research Facts» über Studien im Bereich der Homöopathie, Akupunktur, Antroposophischen Medizin und Phytotherapie ausgewählt und zusammengestellt, die über die Internetseite www.eiccam.eu zugänglich sind. Weitere Publikationen sind in Vorbereitung.

EICCAM verfügt leider erst über bescheidene Spendenmittel. Für die weitere und laufende Tätigkeit werden jedoch wesentlich mehr Mittel benötigt. Interessierte Spender, die dieses wichtige Projekt unterstützen möchten, wenden sich bitte an die Vorsitzende des EICCAM Management Boards, Frau Dr. Susanne Schunder-Tatzber.

Susanne Schunder-Tatzber

$\begin{array}{ll}\text { KARGER } & \text { ๑ 2010 S. Karger GmbH, Freiburg } \\ \text { Fax +4976145207 14 } & \text { Accessible online at: } \\ \text { E-Mail Information@Karger.de } & \text { www.karger.com/szg }\end{array}$

Dr. med. Susanne Schunder-Tatzber, MAS, MBA

Akademie für Ganzheitsmedizin

Otto-Wagner-Spital

Sanatoriumstrasse 2, 1140 Wien, Österreich

Tel. +43 1 688-7507, Fax -750715

schunder@gamed.or.at 